ANNALES

POLONICI MATHEMATICI

$87(2005)$

\title{
The Łojasiewicz exponent of c-holomorphic mappings
}

\author{
by Maciej P. Denkowski (Kraków and Bordeaux)
}

\begin{abstract}
The aim of this paper is to study the Eojasiewicz exponent of c-holomorphic mappings. After introducing an order of flatness for c-holomorphic mappings we give an estimate of the Lojasiewicz exponent in the case of isolated zero, which is a generalization of the one given by Płoski and earlier by Chądzyński for two variables.
\end{abstract}

1. Introduction. Since most of our considerations are of local nature we may place ourselves since the beginning in $\mathbb{C}^{m}$. Let $A \subset \Omega$ be an analytic subset of an open set $\Omega \subset \mathbb{C}^{m}$. We assume that $A$ is a closed set in $\Omega$.

(1.1) Definition ([Ł2], [Wh]). A mapping $f: A \rightarrow \mathbb{C}^{n}$ is called $c$-holomorphic if it is continuous and the restriction of $f$ to the subset $\operatorname{Reg} A$ of regular points of $A$ is holomorphic. We denote by $\mathcal{O}_{\mathrm{c}}\left(A, \mathbb{C}^{n}\right)$ the ring of c-holomorphic mappings, and by $\mathcal{O}_{\mathrm{c}}(A)$ the ring of c-holomorphic functions.

This is a way of generalizing the notion of holomorphicity to sets having singularities. The following theorem motivates this generalization:

(1.2) Theorem ([Wh, 4.5Q]). A mapping $f: A \rightarrow \mathbb{C}^{n}$ is c-holomorphic iff it is continuous and its graph $\Gamma_{f}:=\{(x, f(x)) \mid x \in A\}$ is an analytic subset of $\Omega \times \mathbb{C}^{n}$.

It is worth noting that by a recent result of N. Shcherbina [Sh] the pluripolarity of the graph is sufficient (unlike for instance sub- or semianalyticity: $f(x):=|x|$ for $x \in \mathbb{C}$ has semianalytic graph which is not complex analytic). By (1.2) the zero set of a c-holomorphic function is analytic.

We suppose hereafter that the reader is acquainted with basic properties of c-holomorphic mappings (see [Wh]; c-holomorphic stands in fact for continuous weak holomorphic). We will write briefly "nbhd" for "neighbourhood".

Note that a mapping is c-holomorphic iff all its components are c-holomorphic. If $A$ is analytic and $f \in \mathcal{O}_{\mathrm{c}}(A)$, then it is also easy to see that

2000 Mathematics Subject Classification: 32B15, 32B10, 32B30, 32A17.

Key words and phrases: complex analytic sets, c-holomorphic mappings, Łojasiewicz exponent, regular separation, intersection theory. 
$\operatorname{dim}_{a} A=\operatorname{dim}_{(a, f(a))} \Gamma_{f}$ for $a \in A$. Each regular point $a \in A$ gives a regular point $(a, f(a))$ on the graph. Above a singular point $a \in \operatorname{Sng} A$ one has either a singular point of the graph, or a regular point $b:=(a, f(a))$ for which the tangent space $T_{b} \Gamma_{f}$ contains $\{0\} \times \mathbb{C}$ (in that case see Theorem (1.3) below). Finally, it is clear that a c-holomorphic mapping has an irreducible graph iff its domain is an irreducible set, which is also valid for germs. Many of the theorems concerning holomorphic functions have a straightforward analogue for c-holomorphic functions, at least as long as they do not involve differential structure.

We also assume that the reader is acquainted with the intersection theory as presented in [T2] and [Dr].

We finish this section by giving a characterization of holomorphic germs on an analytic germ. It may be known, but for the convenience of the reader we provide a proof. We denote by $T_{a}^{\mathrm{Zar}} X$ the Zariski tangent space of an analytic germ $X_{a}$ at a point $a \in \mathbb{C}^{m}$ (see [Wh]).

(1.3) Theorem. Let $f \in \mathcal{O}_{\mathrm{c}}\left(A_{a}, \mathbb{C}^{n}\right)$ be a c-holomorphic germ on the analytic germ $A_{a}$ at $a \in \mathbb{C}^{m}$. Then $f$ is holomorphic (i.e. has a holomorphic extension to a nbhd of a in $\left.\mathbb{C}^{m}\right)$ iff $T_{(a, f(a))}^{\mathrm{Zar}} \Gamma_{f} \cap\left(\{0\} \times \mathbb{C}^{n}\right)=\{0\}$.

Before proving this theorem we record the following lemma (we assume submanifolds to be analytic):

(1.4) Lemma. Let $\Gamma \subset \mathbb{C}^{m} \times \mathbb{C}^{n}$ be a $k$-dimensional submanifold with $k \leq m$ and such that $T_{a} \Gamma \cap\left(\{0\} \times \mathbb{C}^{n}\right)=\{0\}$. Then there exists an $m$ dimensional submanifold $\widetilde{\Gamma}$ such that $\widetilde{\Gamma}_{a} \supset \Gamma_{a}$ (as germs at a), $T_{a} \widetilde{\Gamma} \supset T_{a} \Gamma$ and still $T_{a} \widetilde{\Gamma} \cap\left(\{0\} \times \mathbb{C}^{n}\right)=\{0\}$.

One proves this lemma by taking a biholomorphism straightening $\Gamma$ in a nbhd $U \ni a$ and rewriting the condition on the tangent space.

Proof of Theorem (1.3). For simplicity assume that $a=f(a)=0$. For the "if" part take $F \supset f$, a holomorphic extension of $f$ to a nbhd of zero in $\mathbb{C}^{m}$. Taking a submersion describing $\Gamma_{F}$ it is easy to see that $T_{0}^{\text {Zar }} \Gamma_{f} \cap\left(\{0\} \times \mathbb{C}^{n}\right) \subset$ $T_{0} \Gamma_{F} \cap\left(\{0\} \times \mathbb{C}^{n}\right)=\{0\}$.

To prove the "only if" part observe first that the assumption leads to $m \geq \operatorname{dim} T_{0}^{\mathrm{Zar}} \Gamma_{f}$. There exists a submanifold $\Gamma$ such that $\Gamma_{0} \supset\left(\Gamma_{f}\right)_{0}$ and $T_{0} \Gamma=T_{0}^{\mathrm{Zar}} \Gamma_{f}$. By Lemma (1.4) we find an $m$-dimensional submanifold $\widetilde{\Gamma}$ whose germ at zero contains the germ $\Gamma_{0}$ and whose tangent space at zero meets $\{0\} \times \mathbb{C}^{n}$ only at zero. By the implicit function theorem $\widetilde{\Gamma}$ is then the germ of the graph of a holomorphic function over $\mathbb{C}^{m}$.

Note. Example (2.4) in the following section shows that one cannot replace in this theorem the Zariski tangent space by the tangent cone of the graph. 
2. The Łojasiewicz exponent of c-holomorphic maps. The notion of regular separation of closed sets can be found in [Ł1], [T1]. We recall that any two analytic sets $X$ and $Y$ are separated at points $a \in X \cap Y$ with some exponent $\alpha>0$ (depending on the point; we say that $X$ and $Y$ are $\alpha$-separated at $a$ ), i.e. in a nbhd $U \ni a$ we have

$$
\operatorname{dist}(z, X)+\operatorname{dist}(z, Y) \geq c \operatorname{dist}(z, X \cap Y)^{\alpha}
$$

for some $c>0$. We introduce the Lojasiewicz exponent for analytic sets:

$$
\mathcal{L}(X, Y ; a):=\inf \{\alpha>0 \mid X \text { and } Y \text { are } \alpha \text {-separated at } a\}
$$

where $a \in X \cap Y$ and $X, Y$ are analytic subsets of the open set $\Omega \subset \mathbb{C}^{m}$. From the inequality $\operatorname{dist}(z, X)+\operatorname{dist}(z, Y) \leq 2 \operatorname{dist}(z, X \cap Y)$ it follows easily that $\mathcal{L}(X, Y ; a) \geq 1$ if $X$ or $Y$ has empty interior. In the case of an isolated intersection one can find an elegant characterization of this exponent in [T1] (for the general case see $[\mathrm{Cg}],[\mathrm{CgT}]$ ).

We use regular separation to prove the following simple theorem which is basic for our further considerations (we are working in the case $m \geq 1$ ).

(2.1) Theorem. Let $f \in \mathcal{O}_{\mathrm{c}}\left(A, \mathbb{C}^{n}\right)$ with $A \subset \Omega$ and $\Omega \subset \mathbb{C}^{m}$ open. For each $a \in f^{-1}(0)$ the mapping $f$ satisfies condition $(s)$ at the point a, i.e. there exist a nbhd $U$ of a and constants $c, \alpha>0$ such that

$$
|f(x)| \geq c \operatorname{dist}\left(x, f^{-1}(0)\right)^{\alpha}, \quad x \in U \cap A,
$$

where the distance comes from any of the usual norms on $\mathbb{C}^{m}$.

Condition $(*)$ is clearly a condition on germs. If $U$ is small enough to have $\operatorname{dist}\left(x, f^{-1}(0)\right)<1$, then $(*)$ holds for any exponent $\alpha^{\prime} \geq \alpha$. It is therefore natural to study the best exponent, i.e. the Lojasiewicz exponent:

$\mathcal{L}(f ; a):=\inf \{\alpha>0 \mid f$ satisfies condition $(s)$ at $a$ with exponent $\alpha\}$.

If $A_{a}=\bigcup_{j=1}^{r} A^{(j)}$ is the decomposition of the germ $A_{a}$ into irreducible components, then $\mathcal{L}(f ; a) \leq \max _{j=1}^{r} \mathcal{L}\left(\left.f\right|_{A^{(j)}} ; a\right)$, where $\left.f\right|_{A^{(j)}}$ denotes the restriction of $f$ to $A^{(j)}$. If $a$ is isolated in the fibre $f^{-1}(0)$ or, more generally, if the germ $\left(f^{-1}(0)\right)_{a}$ is contained in $\bigcap_{j} A^{(j)}$, then equality holds.

However, the inequality may also be strict: take for instance in $\mathbb{C}^{3}$ the sets $A^{(1)}:=\left\{y^{2}=x^{3}\right\} \times \mathbb{C}, A^{(2)}:=\{y=0\}$ and the c-holomorphic function $f(x, y, z)$ defined to be $y / x$ on $A^{(1)} \backslash\{x=y=0\}$, and 0 on the whole of $A^{(2)}$. Then $\mathcal{L}(f ; 0)=1 / 3, \mathcal{L}\left(\left.f\right|_{A^{(1)}} ; 0\right)=1 / 2$ and obviously $\mathcal{L}\left(\left.f\right|_{A^{(2)}} ; 0\right)=0$.

Note that the Eojasiewicz exponent is a biholomorphic invariant exactly as in the holomorphic case, i.e. for any biholomorphisms $\varphi$ and $\psi$ the mappings $f$ and $\psi \circ f \circ \varphi$ have the same exponent at $a$ and $\varphi^{-1}(a)$ respectively (this permits the notion to be applied on manifolds as well). It does not depend on the norm chosen and one checks that it is intrinsic.

Due to the generalized mean value theorem we obtain: 
(2.2) TheOREM. Let $A$ be an analytic set of pure dimension $k$ in an open set $\Omega \subset \mathbb{C}^{m}$ and let $F: \Omega \rightarrow \mathbb{C}^{n}$ be holomorphic. Let $a \in A$. If $F(a)=0$ and $F$ does not vanish identically on the germ $A_{a}$, then $\mathcal{L}\left(\left.F\right|_{A} ; a\right) \geq 1$.

(2.3) Remark. As already noticed, a c-holomorphic mapping which is not holomorphic can have an exponent smaller than 1 : Indeed, let $A:=$ $\left\{(x, y) \in \mathbb{C}^{2} \mid y^{2}=x^{3}\right\}$ and consider the c-holomorphic function $f(x, y):=$ $y / x$ for $(x, y) \in A, x \neq 0$, and $f(0):=0$. An easy computation leads to $\mathcal{L}(f ; 0)=1 / 2$.

One may wonder whether the fact that the Eojasiewicz exponent is smaller than 1 characterizes "purely" c-holomorphic functions (i.e. non-holomorphic ones). One may hope that at least for an irreducible curve germ $\Gamma$ at $a$ there should be the equivalence: $f \in \mathcal{O}_{\mathrm{c}}(\Gamma) \backslash \mathcal{O}(\Gamma)$ and $f^{-1}(0)=\{a\}$ iff $\mathcal{L}(f ; a)<1$. However, the following example shows that this is not true:

(2.4) Example. Consider the analytic curve $A:=\left\{(x, y) \in \mathbb{C}^{2} \mid y^{3}=x^{4}\right\}$ and the c-holomorphic function $f(x, y):=y^{2} / x$ for $(x, y) \in A, x \neq 0$, and $f(0):=0$. Then $\varphi(t)=\left(t^{3}, t^{4}\right)$ is a Puiseux parametrization of $A$ at zero and $\psi(t)=\left(t^{3}, t^{4}, t^{5}\right)$ a Puiseux parametrization of $\Gamma_{f}$ at zero. It is easy to see that in this case $T_{0}^{\mathrm{Zar}} \Gamma_{f}=\mathbb{C}^{3}$ (while the tangent cone $C_{0}\left(\Gamma_{f}\right)$ is $\mathbb{C} \times\{(0,0)\})$ and so by Theorem $(1.3), f$ is not holomorphic at zero. However, $\mathcal{L}(f ; 0)=5 / 3$ (one may compute this directly as in the previous example or apply Theorem (3.2) proved later on).

We could also ask if for an analytic curve $\Gamma$, irreducible at zero and having a singularity at zero, one could have for $f \in \mathcal{O}_{\mathrm{c}}(\Gamma)$ non-constant, the equivalence $\mathcal{L}(f ; 0)<1$ iff $0 \in \operatorname{Reg} \Gamma_{f}$ and $\{0\} \times \mathbb{C} \subset T_{0} \Gamma_{f}$.

As a matter of fact it suffices to modify slightly the previous example to see that this does not hold either. Take $A=\left\{y^{4}=x^{5}\right\} \subset \mathbb{C}^{2}$ and $f(x, y)=$ $(y / x)^{3}, f(0)=0$. Then $\zeta(t)=\left(t^{4}, t^{5}, t^{3}\right)$ is a Puiseux parametrization of the graph and so $\Gamma_{f}$ has a singularity at zero, but it is also easy to see that $\mathcal{L}(f ; 0)=3 / 4$ (and here also $f$ is not holomorphic).

Nonetheless the converse implication does hold - see end of Section 3.

It may be useful to give a characterization of those c-holomorphic mappings whose exponent is smaller than 1 . This exponent is closely related to the separation exponent of the graph and the domain.

(2.5) Theorem. Suppose that $f \in \mathcal{O}_{\mathrm{c}}\left(A, \mathbb{C}^{n}\right)$ does not vanish identically on $A_{a}$ and let $a \in f^{-1}(0)$. Then

$$
\mathcal{L}(f ; a) \leq \mathcal{L}\left(\Gamma_{f}, A \times\{0\} ;(a, 0)\right) \leq \max \{1, \mathcal{L}(f ; a)\},
$$

which means that the following assertions are then equivalent:

(i) $\mathcal{L}(f ; a) \leq 1$;

(ii) $\mathcal{L}\left(\Gamma_{f}, A \times\{0\} ;(a, 0)\right)=1$. 
If moreover $a$ is isolated in the fibre $f^{-1}(0)$, then the above two conditions are equivalent to the third one (cf. [T1]) concerning the tangent cones:

(iii) $C_{(a, 0)}\left(\Gamma_{f}\right) \cap C_{(a, 0)}(A \times\{0\})=\{(0,0)\}$.

Proof. The equivalence (ii) $\Leftrightarrow($ iii) can be found in [T1, 3.4]. We will show

(a) $\mathcal{L}(f ; a) \leq \mathcal{L}\left(\Gamma_{f}, A \times\{0\} ; a\right)$,

(b) $\mathcal{L}\left(\Gamma_{f}, A \times\{0\} ; a\right) \leq \max \{1, \mathcal{L}(f ; a)\}$.

Let $\alpha \geq \mathcal{L}\left(\Gamma_{f}, A \times\{0\} ; a\right)$ and $l \geq \mathcal{L}(f ; a)$ be good exponents. As in the proof of Theorem (2.1) the regular separation of the graph and $A \times\{0\}$ leads to $|f(x)| \geq$ const $\cdot \operatorname{dist}\left(x, f^{-1}(0)\right)^{\alpha}, x \in U \cap A$. Hence we obtain (a).

On the other hand, we have $(*)$ in a nbhd of $a$. If that nbhd is small enough to have $|f(x)|<1$, we get $\operatorname{dist}\left((x, f(x)), f^{-1}(0) \times\{0\}\right)=\operatorname{dist}\left(x, f^{-1}(0)\right)+|f(x)| \leq \frac{1}{c}|f(x)|^{1 / l}+|f(x)|$, that is,

$$
\left(\frac{c}{c+1} \operatorname{dist}\left((x, f(x)), f^{-1}(0) \times\{0\}\right)\right)^{\max \{1, l\}} \leq|f(x)|,
$$

which in view of Lemma 1.2 from [CgT] (cf. [Ł1, part 18]) gives (b).

Note. As a consequence of $(2.2)$ and (2.3) one has the equality $\mathcal{L}(f ; a)=$ $\mathcal{L}\left(\Gamma_{f},(\Omega \times\{0\}) ;(a, 0)\right)$ for $f \in \mathcal{O}\left(\Omega, \mathbb{C}^{n}\right)$ non-constant in an open set $\Omega$, $f(a)=0$. Note however that in the c-holomorphic case it is not sufficient to compute the Lojasiewicz separation exponent for the graph and $A$ if we want to have complete information about $\mathcal{L}(f ; a)$.

Using the main theorem of [P1] one can easily obtain the rationality and an upper bound for the Łojasiewicz exponent of a c-holomorphic mapping with isolated zeroes. We denote by $m_{0}(g)$ the (geometric) multiplicity at zero of a holomorphic map germ $g:\left(\mathbb{C}^{m}, 0\right) \rightarrow\left(\mathbb{C}^{n}, 0\right)$. When $n=m$ it is defined to be the covering number (multiplicity) $\mu_{0}\left(\left.\pi\right|_{\Gamma_{g}}\right)$ of the branched covering $\left.\pi\right|_{\Gamma_{g}}$ (see [Ch] or [Ł2]) where $\pi(x, y)=y$ and 0 is the unique point in its fibre. If $n \geq m$, we define $m_{0}(g)$ as the improper intersection index $i\left(\Gamma_{g} \cdot\left(\mathbb{C}^{m} \times\{0\}\right) ; 0\right)$ (cf. [ATW]). One checks that $m_{0}(g)=$ $i\left(\Gamma_{g} \cdot\left(\mathbb{C}^{m} \times\{0\}\right) ; 0\right)$ also in the proper intersection case. This definition also makes sense when applied to c-holomorphic mappings having an isolated zero. It is still a biholomorphic invariant (cf. [ATW]). In what follows, $\operatorname{deg}_{a} X$ stands for the classical degree of the analytic set $X$ at a point $a \in X$ (see $[\mathrm{Ch}]$ ).

(2.6) Theorem. Let $A$ be an analytic set of pure dimension $k \geq 1$ in an open set $D \subset \mathbb{C}^{m}$. Let $f \in \mathcal{O}_{\mathrm{c}}\left(A, \mathbb{C}^{n}\right)$ be such that $f^{-1}(0)=\{0\}$. Then 
(i) the greatest lower bound in the definition of $\mathcal{L}(f ; 0)$ is attained and it is of the form $p / q$ with $0<p \leq m_{0}(f), p, q \in \mathbb{N}$;

(ii) $\mathcal{L}(f ; 0) \leq m_{0}(f)-\operatorname{deg}_{0} \Gamma_{f}+1$.

Proof. First we consider the case $n=k$, applying directly the main result of [P1]. We recall a piece of notation from that work:

$$
q_{0}(Z, \pi):=\sup \left\{q \in \mathbb{R} \mid Z \cap(U \times V) \subset\left\{(x, y) \in U \times\left. V|| y|\leq c| x\right|^{q}\right\}\right.
$$

for some nbhd $U \times V$ of zero and a constant $c>0\}$,

where $Z$ is an analytic subset of pure dimension $k$ in a nbhd of zero in $\mathbb{C}^{k} \times \mathbb{C}^{m-k}$ and such that 0 is isolated in the fibre $\pi^{-1}(0) \cap Z$ for the natural projection $\pi(x, y)=x,(x, y) \in \mathbb{C}^{k} \times \mathbb{C}^{m-k}$.

Consider now the natural projection $\pi: D \times \mathbb{C}^{k} \rightarrow \mathbb{C}^{k}$. Observe that $\Gamma_{f}$ is of pure dimension $k$. We shall see that $\mathcal{L}(f ; 0)=1 / q_{0}\left(\Gamma_{f}, \pi\right)$.

Note first that $\Gamma_{f} \cap(D \times\{0\})=\{(0,0)\}$ implies that $\pi$ is proper when restricted to $\Gamma_{f}$ in a nbhd of $0 \in \mathbb{C}^{m}$. We thus have a branched covering and one easily checks that its multiplicity is $i\left(\Gamma_{f} \cdot(D \times\{0\}) ; 0\right)$. The main theorem of [P1] then yields

(i) the supremum in $q_{0}\left(\Gamma_{f}, \pi\right)$ is attained and is of the form $b / a$ with $a, b$ natural, $a \leq i\left(\Gamma_{f} \cdot(D \times\{0\}) ; 0\right)$,

(ii) $q_{0}\left(\Gamma_{f}, \pi\right) \geq \frac{1}{i\left(\Gamma_{f} \cdot(D \times\{0\}) ; 0\right)-\operatorname{deg}_{0} \Gamma_{f}+1}$.

Let now $q>0$ be one of the numbers which are taken into account when computing $q_{0}\left(\Gamma_{f}, \pi\right)$, and $V \times U$ the nbhd and $c>0$ the constant attached to it. We may assume that $V$ and $U$ are such that $f(V) \subset U$. We have $\Gamma_{f} \cap(V \times U) \subset\left\{(x, y) \in V \times\left. U|| x|\leq c| y\right|^{q}\right\}$. That means that

$$
\frac{1}{c}|x|^{1 / q} \leq|f(x)|, \quad x \in V \cap A,
$$

which leads to $\mathcal{L}(f ; 0) \leq 1 / q_{0}\left(\Gamma_{f}, \pi\right)$.

On the other hand, let $\alpha$ be an exponent for $f$, i.e. $|f(x)| \geq \widetilde{c}|x|^{\alpha}$ for $x \in \widetilde{V} \cap A$, where $\widetilde{c}>0$ is a constant and $\widetilde{V}$ a nbhd of $0 \in D$. We get

$$
\Gamma_{f} \cap\left(\widetilde{V} \times \mathbb{C}^{k}\right) \subset\left\{(x, y) \in \widetilde{V} \times\left.\mathbb{C}^{k}|| x\left|\leq \frac{1}{\widetilde{c}}\right| y\right|^{1 / \alpha}\right\} .
$$

Hence $q_{0}\left(\Gamma_{f}, \pi\right) \geq 1 / \mathcal{L}(f ; 0)$.

We now reduce the case $n>k$ to the previous one by the most natural method (see $[\mathrm{S}]$ ). Projecting $\Gamma_{f}$ on $\mathbb{C}^{n}$, the idea is that for the generic linear mapping $\Phi \in \mathrm{L}\left(\mathbb{C}^{n}, \mathbb{C}^{k}\right)$ of rank $k$ we easily obtain $\mathcal{L}(f ; 0) \leq \mathcal{L}(\Phi \circ f ; 0)$. Then for any $\Psi \in \mathrm{L}\left(\mathbb{C}^{n}, \mathbb{C}^{n-k}\right)$ such that $(\Phi, \Psi)$ is an isomorphism it follows that $\mathcal{L}((\Phi, \Psi) \circ f ; 0)=\mathcal{L}(f ; 0)$. An appropriate choice of $\Psi$ gives $\mathcal{L}((\Phi, \Psi) \circ f ; 0)=$ $\mathcal{L}(\Phi \circ f ; 0)$, which ends the proof since $\Phi \circ f$ satisfies the assumptions of the 
first part of our considerations. One checks that $i\left(\Gamma_{\Phi \circ f} \cdot(D \times\{0\}) ; 0\right)=$ $i\left(\Gamma_{f} \cdot(D \times\{0\}) ; 0\right)$ as in [S]. It remains to show that $\operatorname{deg}_{0} \Gamma_{\Phi \circ f}=\operatorname{deg}_{0} \Gamma_{f}$.

Let $L_{\Phi}: \mathbb{C}^{m} \times \mathbb{C}^{n} \ni(x, y) \mapsto(x, \Phi(y)) \in \mathbb{C}^{m} \times \mathbb{C}^{k}$; then $\Gamma_{\Phi \circ f}=L_{\Phi}\left(\Gamma_{f}\right)$. Consider a linear surjection $\Theta: \mathbb{C}^{m} \times \mathbb{C}^{k} \rightarrow \mathbb{C}^{k}$ such that for the generic $z \in \mathbb{C}^{k}$ (small enough) we have $\operatorname{deg}_{0} \Gamma_{\Phi \circ f}=\#\left(\Gamma_{\Phi \circ f} \cap \Theta^{-1}(z)\right)$.

Take then $\Xi:=\Theta \circ L_{\Phi}$ and note that $\operatorname{Ker} \Xi=L_{\Phi}^{-1}(\operatorname{Ker} \Theta)$. So (since it is obvious that $f\left(x_{\nu}\right) \rightarrow 0$ iff $x_{\nu} \rightarrow 0$ )

$$
\begin{aligned}
\text { Ker } \Xi \cap C_{0}\left(\Gamma_{f}\right)=\left\{(x, y) \in \mathbb{C}^{m} \times \mathbb{C}^{n} \mid \exists\left(A \ni x_{\nu} \rightarrow 0\right),\left\{\lambda_{\nu}\right\} \subset \mathbb{C}:\right. \\
\left.\left(\lambda_{\nu} x_{\nu}, \lambda_{\nu} f\left(x_{\nu}\right)\right) \rightarrow(x, y) \text { and } \Theta(x, \Phi(y))=0\right\} .
\end{aligned}
$$

But then for any $(x, y) \in C_{0}\left(\Gamma_{f}\right)$ we have $\lambda_{\nu} \Phi\left(f\left(x_{\nu}\right)\right)=\Phi\left(\lambda_{\nu} f\left(x_{\nu}\right)\right) \rightarrow$ $\Phi(y)$, whence $(x, \Phi(y)) \in C_{0}\left(\Gamma_{\Phi \circ f}\right)$. Since $\Theta(x, \Phi(y))=0$, it follows that $(x, \Phi(y))=(0,0)$. So $y \in \operatorname{Ker} \Phi$. However, $y$ belongs at the same time to the tangent cone at zero of the image of $f$ and because of the choice of $\Phi$ this yields $y=0$.

That means that $\Xi$ realizes the degree $\operatorname{deg}_{0} \Gamma_{f}$, i.e. $\#\left(\Gamma_{f} \cap \Xi^{-1}(z)\right)=$ $\operatorname{deg}_{0} \Gamma_{f}$ for the generic $z \in \mathbb{C}^{k}$ (small enough). Since $L_{\Phi}=\operatorname{id}_{\mathbb{C}^{m}} \times \Phi$, it is clear that $L_{\Phi}^{-1}(x, \Phi(f(x))) \cap \Gamma_{f}=\{(x, f(x))\}$ consists of a single point and so $\mu_{0}\left(\left.L_{\Phi}\right|_{\Gamma_{f}}\right)=1$. Hence $\mu_{0}\left(\left.\Xi\right|_{\Gamma_{f}}\right)=\mu_{0}\left(\left.\Theta\right|_{L_{\Phi}\left(\Gamma_{f}\right)}\right)$, i.e. $\operatorname{deg}_{0} \Gamma_{f}=\operatorname{deg}_{0} \Gamma_{\Phi \circ f}$.

Note. One always has $\operatorname{deg}_{0} \Gamma_{f} \leq \operatorname{deg}_{0} A$ for any $f \in \mathcal{O}_{\mathrm{c}}\left(A, \mathbb{C}^{n}\right)$ with $f(0)=0$ (for convenience). It is easy to check that if $f$ is a restriction of a holomorphic mapping, then equality holds (nonetheless it may hold as well for purely c-holomorphic mappings, cf. Example (2.4)).

We will improve the bound in Section 5. Note that there is a direct way of computing the Łojasiewicz exponent in the case of an isolated zero and $n=k$ (we work under the assumptions of the previous theorem). The number $\mu:=i\left(\Gamma_{f} \cdot\left(D \times \mathbb{C}^{k}\right) ; 0\right)$ is the multiplicity of $f$ at zero. We now follow the idea from [P3] adapting it to our case.

For any c-holomorphic germ $h:(A, 0) \rightarrow(\mathbb{C}, 0)$ we may define the characteristic polynomial of $h$ relative to $f$ by setting, for $y \in \mathbb{C}^{k}$ not critical for $f$ (i.e. $f^{-1}(y)=\left\{x^{1}, \ldots, x^{\mu}\right\}$ consists of $\mu$ distinct points),

$$
P_{h}(y, t):=\prod_{x \in f^{-1}(y)}(t-h(x))=t^{\mu}+a_{1}(y) t^{\mu-1}+\cdots+a_{\mu}(y),
$$

where $a_{j}(y)=(-1)^{j} \sum_{1 \leq i_{1}<\cdots<i_{j} \leq \mu} h\left(x^{i_{1}}\right) \cdots h\left(x^{i_{j}}\right)$ are symmetric polynomials, $a_{j}(0)=0$. It is then clear that there is a small enough nbhd $U$ of $0 \in \mathbb{C}^{k}$ such that $a_{j} \in \mathcal{O}(U \backslash(\sigma \cup f(\operatorname{Sng} A))) \cap \mathcal{C}(U)$, where $\sigma$ is the critical set for $f$ (note that $f$ being proper in a nbhd of zero, the image under $f$ of Sng $A$ in this nbhd is a nowhere dense analytic set in $U$; remember the graph of $f$ over some singular points of $A$ may be regular). Thus $P_{h} \in \mathcal{O}(U)[t]$. It is clear that $P_{h}^{-1}(0)$ is equal to $F(V)$ where $F=(f, h)$ and $V$ is a sufficiently 
small nbhd of zero in $A$. Therefore the inequality $|h(x)| \leq$ const $\cdot|f(x)|^{q}$ in a nbhd of zero is equivalent to the following inclusion of germs at $0 \in \mathbb{C}_{y}^{k} \times \mathbb{C}_{t}$ :

$$
P_{h}^{-1}(0) \subset\left\{(y, t) \in \mathbb{C}^{k} \times \mathbb{C}|| t \mid \leq \text { const } \cdot|y|^{q}\right\} .
$$

Recall now the following crucial lemma of Płoski (see [P1], [P3]):

(2.7) Lemma. If $P(y, t)=t^{\mu}+a_{1}(y) t^{\mu-1}+\cdots+a_{\mu}(y) \in \mathcal{O}_{k}[t]$ is a distinguished polynomial, then $q_{0}(P):=\min _{j=1}^{\mu} \operatorname{ord}_{0}\left(a_{j}\right) / j$ is the largest number $q \in \mathbb{R}$ such that $(\star)$ holds. Moreover $q_{0}(P) \geq 1 /\left(\mu-\operatorname{ord}_{0} P+1\right)$.

If we denote by $O(f, h)$ the least upper bound of all $q>0$ for which $|h(x)| \leq$ const $\cdot|f(x)|^{q}$ in a nbhd of zero in $A$, then it is attained and equal to the number given by Lemma (2.7). Thus it is a rational number. It is easy to see that $\mathcal{L}(f ; 0)=1 / \min _{j=1}^{m} O\left(f, x_{j}\right)$, where $x_{j}: A \rightarrow \mathbb{C}$ are coordinate functions in $\mathbb{C}^{m}$ restricted to $A$.

Observe finally that if $\mathbb{C} v \in G_{1}\left(\mathbb{C}^{k}\right)$ (the first grassmannian, i.e. the collection of all complex lines through 0 in $\left.\mathbb{C}^{k}\right)$, then $S_{v}:=f^{-1}(\mathbb{C} v)$ is a curve germ at zero. The lines $\mathbb{C} v$ such that $\mathbb{C} v \cap\left(\sigma \cup f(\operatorname{Sng} A) \cup \bigcup_{j}\right.$ in $\left.a_{j}^{-1}(0)\right)$ is void or isolated at zero form an open dense subset in $G_{1}\left(\mathbb{C}^{k}\right)$ (here in $a_{j}$ denotes the initial form of $\left.a_{j}\right)$. For such a line, $F\left(S_{v}\right)$ is equal to $\{(y, t) \mid$ $\left.P_{h}(y, t)=0, v_{i} y_{j}=v_{j} y_{i}, i<j\right\}$ and so it is clear that the polynomial for $\left.h\right|_{S_{v}}$ relative to $\left.f\right|_{S_{v}}$ is exactly $\left.P_{h}\right|_{\mathbb{C} v \times \mathbb{C} \text {. Thus the Łojasiewicz exponent }}$ $\mathcal{L}(f ; 0)$ is attained on the generic curve $S_{v}$ (generally not irreducible). Since $\mathcal{L}\left(\left.f\right|_{\Gamma} ; 0\right) \leq \mathcal{L}(f ; 0)$ for any curve germ $\Gamma$ at zero, it now follows that

$$
\mathcal{L}(f ; 0)=\max \left\{\mathcal{L}\left(\left.f\right|_{\Gamma} ; 0\right) \mid \Gamma \text { an irreducible curve germ, } 0 \in \Gamma \subset A\right\} .
$$

3. One-dimensional case. If $A$ is an irreducible analytic curve in an open set $\Omega \subset \mathbb{C}^{m}$ with $0 \in \operatorname{Sng} A$ and $f \in \mathcal{O}_{\mathrm{c}}(A)$ with $f^{-1}(0)=\{0\}$, then we are able to give an explicit formula for $\mathcal{L}(f ; 0)$, which is well known in the holomorphic case. Our proof is similar to the usual one. We shall make use of the Puiseux parametrization of $A$.

First recall the following easy lemma (see [Ch, 1.1.5]):

(3.1) LeMmA. If $f \not \equiv 0$ is a holomorphic function in a nbhd of $0 \in \mathbb{C}^{m}$, $f(0)=0$, and if $\operatorname{ord}_{0} f$ is the order of $f$ at zero, then

$$
\operatorname{ord}_{0} f=\max \left\{\eta>\left.0|\exists c>0:| f(x)|\leq c| x\right|^{\eta} \text { for } x \text { in a nbhd of } 0\right\} .
$$

The explicit formula for $\mathcal{L}(f ; 0)$ in this case is as follows:

(3.2) Theorem. Let $A$ be a one-dimensional locally irreducible analytic subset of an open set $\Omega \subset \mathbb{C}^{m}$ such that $0 \in A$. Let $f \in \mathcal{O}_{\mathrm{c}}\left(A, \mathbb{C}^{n}\right)$ be such that $f^{-1}(0)=\{0\}$. Let $\gamma(t)=\left(t^{d}, \varphi(t)\right)$ be a Puiseux parametrization of $A$ 
in a nbhd of zero (here $d=\operatorname{deg}_{0} A$ and $\left.\operatorname{ord}_{0} \varphi:=\min _{j=1}^{m-1} \operatorname{ord}_{0} \varphi_{j} \geq d\right)$. Then

$$
\mathcal{L}(f ; 0)=\frac{\operatorname{ord}_{0}(f \circ \gamma)}{\operatorname{deg}_{0} A}=\frac{m_{0}(f)}{\operatorname{deg}_{0} A} .
$$

Proof. Since $\lim _{t \rightarrow 0}|\gamma(t)| /|t|^{d}>0$, the separation condition $(*)$ with the best exponent $l_{0}:=\mathcal{L}(f ; 0)$ becomes equivalently $|f(\gamma(t))| \geq$ const $|t|^{d l_{0}}$ for $t$ in a nbhd of zero. By Lemma $(3.1),\left|f_{j}(\gamma(t))\right| \leq\left(\max _{\iota=1}^{n} c_{\iota}\right)|t|^{\min _{\iota=1}^{n} \operatorname{ord}_{0}\left(f_{\iota} \circ \gamma\right)}$ for $t$ in a nbhd of zero. We may assume that $|\cdot|$ is the maximum norm in $\mathbb{C}^{n}$. So we obtain $0<$ const $\leq|t|^{\operatorname{ord}_{0}(f \circ \gamma)-d l_{0}}$ when $t \rightarrow 0$, which is possible only when $l_{0} \geq \operatorname{ord}_{0}(f \circ \gamma) / d$.

Consider the holomorphic function $g:=f \circ \gamma$ and put $r:=\operatorname{ord}_{0} g \geq 1$. If $n=1$ expand $g(t)=\sum_{\nu \geq r} a_{\nu} t^{\nu}$ and fix $0<\varepsilon<1$. For $|t|$ small enough we have

$$
\left|\sum_{\nu=1}^{\infty} \frac{a_{r+\nu}}{a_{r}} t^{\nu}\right| \leq \varepsilon
$$

and so

$$
\left|1+\sum_{\nu=1}^{\infty} \frac{a_{r+\nu}}{a_{r}} t^{\nu}\right| \geq 1-\left|\sum_{\nu=1}^{\infty} \frac{a_{r+\nu}}{a_{r}} t^{\nu}\right| \geq 1-\varepsilon=: c>0 .
$$

Hence $|g(t)| \geq\left|a_{r}\right| \cdot|t|^{r} \cdot c$ for $t$ in a nbhd of zero.

Now if $n>1$, by the previous argument for $t$ in a nbhd of zero we obtain

$$
\left|f_{j}(\gamma(t))\right| \geq c_{j}|t|^{\operatorname{ord}_{0}\left(f_{j} \circ \gamma\right)} \quad \text { for all } j .
$$

We then have, for $t$ in a nbhd of zero,

$$
|g(t)| \geq\left(\min _{j=1}^{n} c_{j}\right)|t|^{\min _{j=1}^{n} \operatorname{ord}_{0} g_{j}}
$$

which is the desired inequality.

In the course of the proof we have shown that for a non-constant holomorphic germ $g:(\mathbb{C}, 0) \rightarrow\left(\mathbb{C}^{n}, 0\right)$ one has the inequalities

$$
c_{1}|t|^{\operatorname{ord}_{0} g} \leq|g(t)| \leq c_{2}|t|^{\operatorname{ord}_{0} g}
$$

near zero, for some $c_{1}, c_{2}>0$. In fact for c-holomorphic mappings we have a result analogous to Lemma (3.1):

(3.3) Proposition. Under the assumptions of Theorem (3.2) one has

$$
|f(x)| \leq \text { const } \cdot|x|^{\operatorname{ord}_{0}(f \circ \gamma) / \operatorname{deg}_{0} A}
$$

for $x$ in a nbhd of zero in $A$, and $\operatorname{ord}_{0}(f \circ \gamma) / \operatorname{deg}_{0} A$ is the maximal exponent with this property.

Proof. On the one hand, $|f(\gamma(t))| \leq$ const $\cdot|t|^{\operatorname{ord}_{0}(f \circ \gamma)}$, while on the other hand $|\gamma(t)| \geq$ const $\cdot|t|^{\operatorname{ord}_{0} \gamma}$ for $t$ in a nbhd of zero. It remains to write down the inequalities starting from $|\gamma(t)|^{\alpha}$, where $\alpha=\operatorname{ord}_{0}(f \circ \gamma) / \operatorname{ord}_{0} \gamma$. 
If there were an exponent $\alpha>\operatorname{ord}_{0}(f \circ \gamma) / \operatorname{ord}_{0} \gamma$ such that $|f(x)| \leq$ const $\cdot|x|^{\alpha}$, then by Theorem (3.2) we would obtain

$$
0<\text { const } \leq|x|^{\alpha-\operatorname{ord}_{0}(f \circ \gamma) / \operatorname{ord}_{0} \gamma}
$$

for $x$ arbitrarily close to zero, hence $\alpha-\operatorname{ord}_{0}(f \circ \gamma) / \operatorname{ord}_{0} \gamma \leq 0$, which is a contradiction.

(3.4) Remark. Combining Theorem (3.2) with Theorem (2.2) we obtain a way of checking whether a given c-holomorphic mapping is holomorphic. Namely, if $A$ is an irreducible analytic set of dimension $k \geq 1$ with $0 \in \operatorname{Sng} A$ and $f \in \mathcal{O}_{\mathrm{c}}\left(A, \mathbb{C}^{n}\right)$, then: if there exists an irreducible one-dimensional germ $\Gamma \subset A$ through zero such that $f^{-1}(0) \cap \Gamma=\{0\}$ and $\mathcal{L}\left(\left.f\right|_{\Gamma} ; 0\right)<1$, then $f$ is "purely" c-holomorphic at zero, i.e. it has no holomorphic extension to any nbhd of zero.

We end this section with an addendum to Example (2.4). Consider $f \in$ $\mathcal{O}_{\mathrm{c}}(\Gamma) \backslash \mathcal{O}(\Gamma)$, where $\Gamma$ is a one-dimensional irreducible analytic germ singular at zero and $f(0)=0$ with 0 regular on the graph. Then $\mathcal{L}(f ; 0) \leq 1$. Indeed, in this case $T_{0} \Gamma_{f}=(\{0\} \times \mathbb{C})$ (cf. introduction), and so there exist sequences $A \ni x_{\nu} \rightarrow 0,\left\{\lambda_{\nu}\right\} \subset \mathbb{C}$ such that $\lambda_{\nu} x_{\nu} \rightarrow 0$ and $\lambda_{\nu} f\left(x_{\nu}\right) \rightarrow v \neq 0$. Now we apply Proposition (3.3) to get, for some constant $c>0$ and $\nu$ large enough,

$$
\left|\lambda_{\nu} f\left(x_{\nu}\right)\right| \leq c\left|\lambda_{\nu}\right|\left|x_{\nu}\right|^{l_{0}}=c\left|\lambda_{\nu} x_{\nu}\right|\left|x_{\nu}\right|^{l_{0}-1}
$$

where $l_{0}=\mathcal{L}(f ; 0)$. Thus $l_{0} \leq 1$. Example $(2.4)$ shows that the converse implication does not hold.

4. The order of flatness of a c-holomorphic function. In the next section we prove a generalization of the estimate of the Lojasiewicz exponent for holomorphic mappings given in [P2], [P3] (by [S] this result still holds when the target space is of dimension $n \geq m$ ).

In our case there is the problem of defining the order of vanishing for a c-holomorphic function since we have no differential structure to rely on. In our definition we will make use of an inequality analogous to the one appearing in Lemma (3.1).

We assume that the analytic germ $A \subset \mathbb{C}_{z}^{k} \times \mathbb{C}_{w}^{m-k}$ under consideration (with $x=(z, w)$ ) is pure $k$-dimensional and such that the projection $\pi(z, w)=z$ is proper on it and $\pi^{-1}(0) \cap C_{0}(A)=\{0\}$. Then $\pi$ is a branched covering on $A$ with covering number $d:=\operatorname{deg}_{0} A$ and a critical set $\sigma$.

Now, whenever $x \notin \sigma$, we may define for a non-constant c-holomorphic germ $f:(A, 0) \rightarrow(\mathbb{C}, 0)$ the polynomial

$$
P(x, t)=\prod_{x^{\prime} \in \pi^{-1}(\pi(x)) \cap A}\left(t-f\left(x^{\prime}\right)\right) .
$$


Since $f$ is holomorphic on $\operatorname{Reg} A$ and continuous, we obtain a distinguished Weierstrass polynomial of degree $d$ with holomorphic coefficients, $P(x, t)=$ $t^{d}+a_{1}(x) t^{d-1}+\cdots+a_{d}(x)$, such that $P(x, f(x))=0$ for $x \in A$. Note that $P$ does not depend on $w$. With this notation we obtain

(4.1) Proposition. If $f:(A, 0) \rightarrow(\mathbb{C}, 0)$ is a non-constant c-holomorphic germ on a pure $k$-dimensional analytic germ at $0 \in \mathbb{C}^{m}$, then the least upper bound of the exponents $\eta>0$ for which there exists a constant $c>0$ such that $|f(x)| \leq c|x|^{\eta}$ in a nbhd of zero is equal to $q_{0}(P)=$ $\min _{j=1}^{d} \operatorname{ord}_{0}\left(a_{j}\right) / j$. It is thus attained and is a positive rational number, independent of the projection $\pi$ chosen (satisfying $\pi^{-1}(0) \cap C_{0}(A)=\{0\}$ ).

Proof. We shall apply [P1]. Since $f(x)$ is a root of the polynomial $P(x, \cdot)$, we have $|f(x)| \leq 2 \max _{j=1}^{d}\left|a_{j}(x)\right|^{1 / j}$ and since $a_{j}$ are holomorphic, for each $j$ we have $\left|a_{j}(x)\right| \leq c_{j}|x|^{\operatorname{ord}_{0} a_{j}}$ near zero (for some constant $c_{j}$, cf. Lemma (3.1), because $a_{j}(0)=0$ ). Hence there exists a constant $c>0$ such that $|f(x)| \leq c|x|^{q_{0}(P)}$ in a nbhd of zero.

Observe now that the projection $\rho=\pi \times \mathrm{id}_{\mathbb{C}}$ is proper on $\Gamma_{f}$ near zero and so $\Gamma:=\rho\left(\Gamma_{f}\right)$ is analytic of pure dimension $k$. The projection $\zeta(z, t)=z$, $(z, t) \in \mathbb{C}^{k} \times \mathbb{C}$, is proper on $\Gamma$ in a nbhd of zero. We may then apply the main result of [P1] to get the inclusion of germs $\Gamma \subset\left\{\left.(z, t)|| t|\leq c| z\right|^{q}\right\}$ for some constants $c, q>0$. This yields $|f(z, w)| \leq c|z|^{q}$ and assuming for instance that $|(z, w)|=|z|+|w|$, we obtain $|f(z, w)| \leq c|(z, w)|^{q}$ for $(z, w) \in A$ in a nbhd of zero. Thus the least upper bound of exponents good for $f$ cannot be smaller than $q_{0}(\Gamma, \zeta)$ (we adopt the notation from the proof of Theorem (2.6), cf. $[\mathrm{P} 1])$.

On the other hand, if, for some $c, \eta>0,|f(x)| \leq c|x|^{\eta}$ in a nbhd of zero, then obviously $|f(z, w)|^{1 / \eta} \leq c^{1 / \eta}(|z|+|w|)$. But since $\pi^{-1}(0) \cap C_{0}(A)=\{0\}$, we have $q_{0}(A, \pi) \geq 1$ (cf. [P1]) and so $|w| \leq c^{\prime}|z|$ for $(z, w) \in A$ in a nbhd of zero and for a constant $c^{\prime}>0$. Thus $|f(z, w)| \leq c\left(1+c^{\prime}\right)^{\eta}|z|^{\eta}$ for $z$ in a nbhd of zero and $w$ such that $(z, w) \in A$, which means that $q_{0}(\Gamma, \zeta) \geq \eta$. Hence the least upper bound sought is actually equal to $q_{0}(\Gamma, \zeta)$ and since $q_{0}(\Gamma, \zeta)=q_{0}(P)$ by Lemma (2.7) (cf. [P1], [P3]) we obtain the final result.

We are now able to state the following definition:

(4.2) Definition. Let $f:(A, 0) \rightarrow(\mathbb{C}, 0)$ be a non-constant c-holomorphic germ on a pure dimensional analytic germ $A$ at zero. We define the order of flatness of $f$ at zero to be

$$
\operatorname{ord}_{0} f:=\max \left\{\eta>0|| f(x) \mid \leq \text { const } \cdot|x|^{\eta} \text { in a nbhd of } 0\right\} .
$$

We put by definition $\operatorname{ord}_{0} 0:=+\infty$.

It is clear that if $A$ is an open subset of $\mathbb{C}^{m}$ we get the usual order of vanishing of a holomorphic function at a point. If $A_{0}=\bigcup_{j=1}^{r} A^{(j)}$ is the 
decomposition of the germ $A_{0}$ into irreducible components, then $\operatorname{ord}_{0} f=$ $\min _{j} \operatorname{ord}_{0}\left(\left.f\right|_{A^{(j)}}\right)$.

One easily checks that $\operatorname{ord}_{0} f$ is a biholomorphic invariant. It is obvious that it does not depend on the norm chosen and it is also clear that it is intrinsic. If $f$ is a mapping, Definition (4.2) also makes sense since it is easy to check that then $\operatorname{ord}_{0} f=\min _{j=1}^{n} \operatorname{ord}_{0} f_{j}$.

For a non-constant c-holomorphic map germ $g:(X, 0) \rightarrow(A, 0)$ one obviously has the inequality $\operatorname{ord}_{0}(f \circ g) \geq \operatorname{ord}_{0} f \cdot \operatorname{ord}_{0} g$ and it is also easy to see that for another non-constant c-holomorphic germ $h:(A, 0) \rightarrow(\mathbb{C}, 0)$,

$\operatorname{ord}_{0}(f+h) \geq \min \left\{\operatorname{ord}_{0} f, \operatorname{ord}_{0} h\right\} \quad$ and $\quad \operatorname{ord}_{0}(f h) \geq \operatorname{ord}_{0} f+\operatorname{ord}_{0} h$ (the last inequality may be strict: take e.g. $A=\left\{x^{3}=y^{2}\right\} \cup\left\{x^{2}=y^{3}\right\}$ in $\mathbb{C}^{2}$ and $f(x, y)=x, g(x, y)=y)$. Moreover, $\operatorname{ord}_{0} f^{q}=q \cdot \operatorname{ord}_{0} f$ for an integer $q>0$.

In the previous section (cf. (3.2) and (3.3)) we showed that for a curve $\Gamma$ irreducible at zero and $f \in \mathcal{O}_{\mathrm{c}}\left(\Gamma, \mathbb{C}^{n}\right)$ with 0 isolated in $f^{-1}(0)$,

$$
m_{0}(f)=\operatorname{ord}_{0} f \cdot \operatorname{deg}_{0} \Gamma \text { and } \mathcal{L}(f ; 0)=\operatorname{ord}_{0} f .
$$

Taking the function $f$ from Example (2.3) one sees that the order of a cholomorphic function may be smaller than 1 .

Note also that $\operatorname{ord}_{0} f$ may not be an integer even when $f$ is the restriction of a holomorphic function (e.g. $\operatorname{ord}_{0}\left(\left.y\right|_{\left\{y^{2}=x^{3}\right\}}\right)=3 / 2$ ) though obviously in this case $\operatorname{ord}_{0} f \geq 1$. In general, by (4.1) we only know that $\left(\operatorname{deg}_{0} A\right) ! \cdot \operatorname{ord}_{0} f \in \mathbb{N}$.

Finally, by Lemma (2.7) we also have

$$
\operatorname{ord}_{0} f \geq \frac{1}{\operatorname{deg}_{0} A-\operatorname{ord}_{0} P+1},
$$

where $P$ is the polynomial from Proposition (4.1). The latter yields:

(4.3) Corollary. Under the assumptions of Proposition (4.1) and with its notation, $\operatorname{ord}_{0} f \geq 1$ iff $C_{0}\left(\Gamma_{f}\right) \cap(\{0\} \times \mathbb{C})=\{0\}$.

Proof. Observe that $\operatorname{ord}_{0} f \geq 1$ iff $\operatorname{ord}_{0} a_{j} \geq j$ for all $j=1, \ldots, d$. It is easily seen that the latter is equivalent to $\operatorname{ord}_{0} P=d$.

We also have $C_{0}\left(P^{-1}(0)\right)=$ in $P^{-1}(0)$ and the condition $\operatorname{ord}_{0} a_{j} \geq j$ for all $j$ is equivalent to $C_{0}(\Gamma) \cap(\{0\} \times \mathbb{C})=\{0\}$ (where $\Gamma=P^{-1}(0) \cap$ $\{w=0\}$ is exactly the image of $\Gamma_{f}$ under the projection $\rho$ from the proof of Proposition (4.1)). This in turn is equivalent to $C_{0}\left(\Gamma_{f}\right) \cap(\{0\} \times \mathbb{C})=\{0\}$.

In the holomorphic setting we may compute the order of vanishing using restriction to a generic complex line. We have a similar possibility in the c-holomorphic case:

(4.4) Proposition. Under the assumptions of the previous proposition there exists an open dense set $U$ in $G_{1}\left(\mathbb{C}^{k}\right)$ such that $\operatorname{ord}_{0} f=\left.\operatorname{ord}_{0} f\right|_{S_{l}}$, 
where $S_{l}:=\pi^{-1}(l)$ for $l \in U$. Then if $S_{l}=\bigcup_{j=1}^{r} S^{(j)}$ is the decomposition of the germ $S_{l}$ into irreducible components $\left(r=r(l) \leq d=\operatorname{deg}_{0} A\right)$ and $\gamma_{j}$ denotes the Puiseux parametrization of the jth component, we have

$$
\operatorname{ord}_{0} f=\min _{j=1}^{r} \frac{\operatorname{ord}_{0}\left(f \circ \gamma_{j}\right)}{\operatorname{ord}_{0} \gamma_{j}} \quad \text { and } \quad d=\sum_{j=1}^{r} \operatorname{ord}_{0} \gamma_{j} .
$$

Proof. This follows directly from the formula for $\operatorname{ord}_{0} f$ and the fact that $\operatorname{ord}_{0} a_{j}$ is attained on the generic line. More precisely, if $l \in G_{1}\left(\mathbb{C}^{k}\right)$ is such that $\operatorname{dim}\left[l \cap\left(\sigma \cup \bigcup_{j=1}^{d}\right.\right.$ in $\left.\left.a_{j}^{-1}(0)\right)\right] \leq 0$, where $\sigma$ is the critical set of the branched covering $\left.\pi\right|_{A}$, then $S_{l}$ is a pure one-dimensional set with covering number $d$ over $l$. What is more, the polynomial used to compute $\left.\operatorname{ord}_{0} f\right|_{S_{l}}$ is $\left.P\right|_{l \times \mathbb{C}}$. Therefore $\operatorname{ord}_{0} f=\min _{j=1}^{d} \operatorname{ord}_{0}\left(\left.a_{j}\right|_{l}\right) / j=\operatorname{ord}_{0}\left(\left.f\right|_{S_{l}}\right)$ and so the result follows by decomposing $S_{l}$ into irreducible germs and applying Theorem (3.2).

This proposition is most interesting when $S_{l}$ is irreducible or if the number of irreducible components of $S_{l}$ is exactly $\operatorname{deg}_{0} A$.

(4.5) COROLlaRY. Under the assumptions of the previous proposition and with its notation,

(i) if for some $l \in U$ the curve germ $S_{l}$ is irreducible, then $\operatorname{ord}_{0} f=$ $\operatorname{ord}_{0}(f \circ \gamma) / \operatorname{deg}_{0} A$, where $\gamma$ is the Puiseux parametrization of $S_{l}$;

(ii) if for some $l \in U$ the curve germ $S_{l}$ has $\operatorname{deg}_{0} A$ irreducible components, then $\operatorname{ord}_{0} f$ is a positive integer.

Since for any curve germ $\Gamma \subset A$ irreducible at zero one has $\operatorname{ord}_{0}\left(\left.f\right|_{\Gamma}\right) \geq$ $\operatorname{ord}_{0} f$, it is clear that by the previous results

$\operatorname{ord}_{0} f=\min \left\{\operatorname{ord}_{0}\left(\left.f\right|_{\Gamma}\right) \mid \Gamma\right.$ an irreducible curve germ, $\left.0 \in \Gamma \subset A\right\}$.

We also have the following counterpart of a known lemma (cf. [Ch]):

(4.6) Proposition. Let $f:(A, 0) \rightarrow\left(\mathbb{C}^{k}, 0\right)$ be a c-holomorphic germ with $f^{-1}(0)=\{0\}$ on a pure $k$-dimensional analytic germ $A$. Then

$$
m_{0}(f) \geq \operatorname{deg}_{0} A \prod_{j=1}^{k} \operatorname{ord}_{0} f_{j} .
$$

Proof. We will adapt to our case the proof from [P2] inspired by P. Tworzewski. We assume without loss of generality that $A \subset \mathbb{C}_{z}^{m}=\mathbb{C}_{x}^{k} \times \mathbb{C}_{y}^{m-k}$ is such that $\pi^{-1}(0) \cap C_{0}(A)=\{0\}$ for $\pi(x, y)=x$. Then the covering number of the branched covering $\left.\pi\right|_{A}$ is $d:=\operatorname{deg}_{0} A$. Let $q_{j}$ denote the denominator of $\operatorname{ord}_{0} f_{j}$ and for simplicity write $F_{j}:=f_{j}^{q_{j}}$. Then $m_{0}(F)=m_{0}(f) \prod_{j} q_{j}$ and $\operatorname{ord}_{0} F_{j}=q_{j} \operatorname{ord}_{0} f_{j}$. 
Consider the set

$$
G:=\left\{(z, w) \in A \times \mathbb{C}^{k} \mid P_{j}\left(z, w_{j}\right)=0, j=1, \ldots, k\right\},
$$

where $P_{j}(z, t):=t^{\operatorname{ord}_{0} F_{j}}-F_{j}(z) \in \mathcal{O}_{\mathrm{c}}(A)[t]$ and so $G$ is analytic. It is obvious that the natural projection $\zeta(z, w)=z$ is proper on $G$ and so is $\left.(\pi \circ \zeta)\right|_{G}$ in a nbhd of zero. It is clearly an $s$-sheeted analytic covering with $s=d \prod_{j} \operatorname{ord}_{0} F_{j}$ (and so $G$ has pure dimension $k$, cf. [Ch, p. 47]).

Take now $v \in \mathbb{C}^{k}$ such that $(0, v) \in C_{0}(G)$ and let $\lambda_{\nu}, z_{\nu}, w_{\nu}$ be the sequences for computing $(0, v)$. Since $\lambda_{\nu} w_{\nu, j} \rightarrow v_{j}$ and since in view of the inequality $\left|F_{j}\left(z_{\nu}\right)\right| \leq c_{j}\left|z_{\nu}\right|^{\operatorname{ord}_{0} F_{j}}$ for some constant $c_{j}>0$ and $\nu \gg 1$, one has $\left|\lambda_{\nu} w_{\nu, j}\right| \leq$ const $\cdot\left|\lambda_{\nu} z_{\nu}\right|$, it follows that $v_{j}=0$. We have thus shown that $(\pi \circ \zeta)^{-1}(0) \cap C_{0}(G)=\{0\}$, which means that

$$
\operatorname{deg}_{0} G=\mu_{0}\left(\left.(\pi \circ \zeta)\right|_{G}\right)=d \prod_{j} \operatorname{ord}_{0} F_{j} .
$$

On the other hand, one easily sees that the projection $\varrho(z, w)=w$ is proper on $G$ in a nbhd of zero and has covering number $m_{0}(F)$. Thus $m_{0}(F) \geq d \prod_{j} \operatorname{ord}_{0} F_{j}$, which yields the desired inequality.

Note. One checks exactly as in [S] that this result is also valid when the target space is of dimension $n>k$. In this case the estimate is exactly the same (i.e. the product still consists of $k$ factors) if $f_{j}$ are ordered in such a way that $\operatorname{ord}_{0} f_{1} \leq \cdots \leq \operatorname{ord}_{0} f_{n}$.

One can also easily prove (using (2.5) and (3.2)) the following

(4.7) Proposition. Let $f \in \mathcal{O}_{\mathrm{c}}\left(A, \mathbb{C}^{n}\right)$ be such that $f^{-1}(0)=\{0\}$. Then

$$
\min \left\{1, \operatorname{ord}_{0} f\right\} \leq \frac{1}{\mathcal{L}\left(\Gamma_{f},\{0\} \times \mathbb{C}^{n} ; 0\right)} \leq \mathcal{L}(f ; 0) .
$$

Thus $C_{0}\left(\Gamma_{f}\right) \cap\left(\{0\} \times \mathbb{C}^{n}\right)=\{0\}$ implies $\mathcal{L}(f ; 0) \geq 1$. Moreover, if $A_{0}$ is an irreducible curve germ, then

$$
\mathcal{L}(f ; 0) \leq 1 \quad \text { implies } \quad \mathcal{L}(f ; 0)=\frac{1}{\mathcal{L}\left(\Gamma_{f},\{0\} \times \mathbb{C}^{n} ; 0\right)} .
$$

We finish this section with an estimate of the Łojasiewicz exponent in the general case. Consider $f \in \mathcal{O}_{\mathrm{c}}\left(A, \mathbb{C}^{n}\right)$ non-constant and such that $f(0)=0$. Then by Theorem (2.1), $f$ satisfies condition $(s)$ at zero. We suppose hereafter that $\operatorname{dim}_{0} A \geq 1$.

(4.8) Lemma. If $f$ is as above and $\Gamma \subset A$ is any analytic curve irreducible at zero such that $C_{0}(\Gamma) \cap C_{0}\left(f^{-1}(0)\right)=\{0\}$, then $\mathcal{L}(f ; 0) \geq \mathcal{L}\left(\left.f\right|_{\Gamma} ; 0\right)$. Thus also $\mathcal{L}(f ; 0) \geq \operatorname{ord}_{0} f$.

Proof. We may suppose that $A$ is of constant dimension. Note first that curves satisfying the assumptions always exist. On the one hand, condition 
$(s)$ on $\Gamma$ yields $|f(\gamma(t))| \geq c_{1} \operatorname{dist}\left(\gamma(t), f^{-1}(0)\right)^{\alpha}$ for $t$ in a nbhd of zero, where $\gamma$ is a Puiseux parametrization of $\Gamma$ at zero. On the other hand, by [T1, 3.4], $\Gamma$ and $f^{-1}(0)$ are 1-separated at zero since $\Gamma$ is transversal to the zero set of $f$. Thus we obtain $|f(\gamma(t))| \geq$ const $\cdot|\gamma(t)|^{\alpha}$, whence the first asserted inequality follows. The second inequality follows from (3.2) and (3.3).

By this lemma, the beautiful result of Cygan $([\mathrm{Cg}])$ and Theorem (2.5) we have the following straightforward relationship between $\mathcal{L}(f ; 0)$ and the degree $\operatorname{deg}_{0}\left(\Gamma_{f} \bullet(A \times\{0\})\right)$ of the improper intersection cycle $\Gamma_{f} \bullet(A \times\{0\})$ in the sense of Tworzewski (see [T2] for the definition and properties).

(4.9) ThEOREM. If $f \in \mathcal{O}_{\mathrm{c}}\left(A, \mathbb{C}^{n}\right)$ is non-constant and such that $f(0)=0$, then $\operatorname{ord}_{0} f \leq \mathcal{L}(f ; 0) \leq \operatorname{deg}_{0}\left(\Gamma_{f} \bullet(A \times\{0\})\right)$.

5. A better estimate of the Łojasiewicz exponent. Now we are ready to prove an addendum to Theorem (2.6). To do this we generalize a lemma and a proposition due to Płoski (cf. [P2]). In the following, $0 \notin \mathbb{N}$ (by definition). Recall that for a pure $k$-dimensional locally analytic set $X$ with proper projection $\pi$ onto $\mathbb{C}^{k}$ and such that $\pi^{-1}(0) \cap X=\{0\}$ we define, for any $h \in \mathcal{O}_{m}$, the characteristic polynomial $P_{h}$ relative to $\left.\pi\right|_{X}$ by setting $P_{h}(x, t)=\prod_{z \in \pi^{-1}(x) \cap X}(t-h(z))$ if $x \notin \sigma, \sigma$ being the critical set for $\pi$, and then extending the coefficients through $\sigma$ by Riemann's theorem. We have the following

(5.1) Lemma ([P2, (2.2)]). In the above setting, for any linear form $L \in$ $\mathrm{L}\left(\mathbb{C}^{m}, \mathbb{C}\right)$ one has $\operatorname{ord}_{0} P_{L} \geq \operatorname{deg}_{0} X$.

We then obtain the following counterpart of [P2, (2.3)]:

(5.2) Proposition. Let $f:(A, 0) \rightarrow\left(\mathbb{C}^{k}, 0\right)$ be a c-holomorphic germ on a pure $k$-dimensional germ $A \subset \mathbb{C}^{m}$ at zero such that $f^{-1}(0)=\{0\}$, and let $g:(A, 0) \rightarrow(\mathbb{C}, 0)$ be a non-constant c-holomorphic function germ. Let $q$ and $q_{j}$ be any positive integers such that $q \operatorname{ord}_{0} g, q_{j} \operatorname{ord}_{0} f_{j} \in \mathbb{N}$. Finally, let $P_{G} \in \mathcal{O}_{k}[t]$ be the characteristic polynomial of $G:=g^{q}$ relative to $F:=\left(f_{1}^{q_{1}}, \ldots, f_{k}^{q_{k}}\right)$ (i.e. relative to $\left.\pi\right|_{\Gamma_{F}}$, where $\pi$ is the natural projection onto $\left.\mathbb{C}^{k}\right)$. Then, if $w \in \mathbb{C}^{k}$,

$$
\begin{aligned}
\operatorname{ord}_{0} P_{G}\left(w_{1}^{q_{1} \operatorname{ord}_{0} f_{1}}, \ldots, w_{k}^{q_{k} \operatorname{ord}_{0} f_{k}},\right. & \left.t^{q \operatorname{ord}_{0} g}\right) \\
& \geq \operatorname{deg}_{0} A \operatorname{ord}_{0} g \prod_{j=1}^{k} \operatorname{ord}_{0} f_{j} \cdot q \prod_{j=1}^{k} q_{j} .
\end{aligned}
$$

Proof. Let $\Gamma$ be the graph of $(F, G)$. It is a pure $k$-dimensional analytic germ at zero with proper projection $\varrho: \mathbb{C}^{m} \times \mathbb{C}^{k} \times \mathbb{C} \ni(z, w, t) \mapsto w \in \mathbb{C}^{k}$. It is clear that for any $w$ which is not critical for $\left.\varrho\right|_{\Gamma}$ or for $F$, one has exactly 
$m_{0}(f) \prod_{j} q_{j}=m_{0}(F)$ points in the fibre $\varrho^{-1}(w) \cap \Gamma$. It is thus the covering number $\mu_{0}\left(\left.\varrho\right|_{\Gamma}\right)$.

Take $L(z, w, t)=t$ and let $P_{L} \in \mathcal{O}_{k}[t]$ be its characteristic polynomial relative to $\left.\varrho\right|_{\Gamma}$. One easily sees that $P_{L}=P_{G}$. If now

$\widetilde{\Gamma}:=\left\{(z, w, t) \in A \times \mathbb{C}^{k} \times \mathbb{C} \mid g(z)^{q}=t^{q \operatorname{ord}_{0} g}, f_{j}(z)^{q_{j}}=w_{j}^{q_{j} \operatorname{ord}_{0} f_{j}}, j=1, \ldots, k\right\}$, then it is easy to see that $\mu_{0}\left(\left.\varrho\right|_{\Gamma}\right)=m_{0}(F) q \operatorname{ord}_{0} g$. On the other hand, if $\zeta$ is a projection in $\mathbb{C}^{m}$ realizing $\operatorname{deg}_{0} A$ (i.e. $\zeta^{-1}(0) \cap C_{0}(A)=\{0\}$ ) and if $p(z, w, t)=z$, then one checks exactly as in the proof of Proposition (4.6) that $(\zeta \circ p)^{-1}(0) \cap C_{0}(\widetilde{\Gamma})=\{0\}$ and so

$$
\operatorname{deg}_{0} \widetilde{\Gamma}=\mu_{0}\left(\left.(\zeta \circ p)\right|_{\widetilde{\Gamma}}\right)=\operatorname{deg}_{0} A q \operatorname{ord}_{0} g \prod_{j=1}^{k} q_{j} \operatorname{ord}_{0} f_{j} .
$$

Finally, consider the characteristic polynomial $\widetilde{P}_{L}$ of $L$ relative to $\left.\varrho\right|_{\widetilde{\Gamma}}$. Once again it is easy to observe that $\widetilde{P}_{L}(w, t)=P_{L}\left(w_{1}^{q_{1} \operatorname{ord}_{0} f_{1}}, \ldots, w_{k}^{q_{k}} \operatorname{ord}_{0} f_{k}\right.$, $\left.t^{q \operatorname{ord}_{0} g}\right)$. Hence we obtain

$$
\operatorname{ord}_{0} P_{G}\left(w_{1}^{q_{1} \operatorname{ord}_{0} f_{1}}, \ldots, w_{k}^{q_{k} \operatorname{ord}_{0} f_{k}}, t^{q \operatorname{ord}_{0} g}\right)=\operatorname{ord}_{0} \widetilde{P}_{L} \geq \operatorname{deg}_{0} \widetilde{\Gamma}
$$

by Lemma (5.1), which is the required inequality.

Similarly to [P2] we obtain

(5.3) Corollary. If $f:(A, 0) \rightarrow\left(\mathbb{C}^{k}, 0\right)$ is a c-holomorphic germ on a pure $k$-dimensional analytic germ $A$ such that $f^{-1}(0)=\{0\}$, then for any c-holomorphic germ $g:(A, 0) \rightarrow(\mathbb{C}, 0)$ there are $k$ functions $h_{j} \in \mathcal{O}_{\mathrm{c}}(A)$ such that in a nbhd of zero $g^{q \cdot m_{0}(f) \prod_{j} q_{j}}=\sum_{j=1}^{k} h_{j} f_{j}^{q_{j}}$ and

$$
q_{j} \operatorname{ord}_{0} f_{j}+\operatorname{ord}_{0} h_{j} \geq \operatorname{deg}_{0} A q \operatorname{ord}_{0} g \prod_{i=1}^{k} q_{i} \operatorname{ord}_{0} f_{i} \quad \text { for } j=1, \ldots, k,
$$

where $q, q_{j} \in \mathbb{N}$ are such that $q \operatorname{ord}_{0} g, q_{j} \operatorname{ord}_{0} f_{j} \in \mathbb{N}$.

Proof. Set $F:=\left(f_{1}^{q_{1}}, \ldots, f_{k}^{q_{k}}\right)$ and $G:=g^{q}$. The characteristic polynomial $P_{G} \in \mathcal{O}_{k}[t]$ is unitary of degree $m_{0}(f) \prod_{j} q_{j}=m_{0}(F)$ and distinguished. Therefore applying Hadamard's lemma to its coefficients $a_{j}$ we have $a_{j}(w)=\sum_{i=1}^{k} w_{i} a_{j}^{i}(w)$, and so one may write $P_{G}$ as

$$
P_{G}(w, t)=t^{m_{0}(F)}+w_{1} H_{1}(w, t)+\cdots+w_{k} H_{k}(w, t),
$$

where $H_{j}(w, t)=\sum_{l=1}^{m_{0}(F)} a_{l}^{j}(w) t^{m_{0}(F)-l} \in \mathcal{O}_{k}[t] \subset \mathbb{C}\{y, t\}$. Without loss of generality we may assume that the supports of the series of $w_{j} H_{j}(w, t)$ are pairwise disjoint, and so if we write

$$
v(h):=\operatorname{ord}_{0} h\left(w_{1}^{q_{1} \operatorname{ord}_{0} f_{1}}, \ldots, w_{k}^{q_{k} \operatorname{ord}_{0} f_{k}}, t^{q \operatorname{ord}_{0} g}\right) \quad \text { for } h \in \mathcal{O}_{k+1},
$$

we have $v\left(w_{j} H_{j}(w, t)\right) \geq v\left(P_{G}(w, t)\right)$ for $j=1, \ldots, k$. Now we require only 
Lemma. If $h:(X, 0) \rightarrow\left(\mathbb{C}_{y}^{n}, 0\right)$ and $P:\left(\mathbb{C}_{y}^{n}, 0\right) \rightarrow(\mathbb{C}, 0)$ are non-constant c-holomorphic germs, where $X \subset \mathbb{C}_{x}^{m}$ is an analytic germ at zero, then

$$
\operatorname{ord}_{0} P(h(x)) \cdot \max _{j=1}^{n} r_{j} \geq \operatorname{ord}_{0} P\left(y_{1}^{r_{1}} \operatorname{ord}_{0} h_{1}, \ldots, y_{n}^{r_{n} \operatorname{ord}_{0} h_{n}}\right),
$$

where $r_{j}$ are any positive integers such that $r_{j} \operatorname{ord}_{0} h_{j} \in \mathbb{N}$.

Proof of the lemma. Let $\theta:=\operatorname{ord}_{0} P\left(y_{1}^{r_{1} \operatorname{ord}_{0} h_{1}}, \ldots, y_{n}^{r_{n}} \operatorname{ord}_{0} h_{n}\right)$. Then there is a polydisc $V$ of radius $R<1$ such that

$$
\left|P\left(y_{1}^{r_{1} \operatorname{ord}_{0} h_{1}}, \ldots, y_{n}^{r_{n} \operatorname{ord}_{0} h_{n}}\right)\right| \leq \text { const } \cdot|y|^{\theta}, \quad y \in V .
$$

We take a nbhd $U$ of $0 \in \mathbb{C}^{m}$ such that $h(U \cap X) \subset V$ and for each $x \in U \cap X$ we choose $y$ so that $y_{j}^{r_{j}} \operatorname{ord}_{0} h_{j}=h_{j}(x)$ for $j=1, \ldots, n$. Then we obtain $|P(h(x))| \leq$ const $\cdot \max \left\{\left|h_{1}(x)\right|^{\frac{1}{r_{1} \text { ord }_{0} h_{1}}}, \ldots,\left|h_{n}(x)\right|^{\frac{1}{r_{n} \text { ord }_{0} h_{n}}}\right\}^{\theta}, \quad x \in U \cap X$.

Shrinking $U$ if necessary, we may assume that $|x|<1$ and $\left|h_{j}(x)\right| \leq$ const . $|x|^{\operatorname{ord}_{0} h_{j}}$ for all $j$ and $x \in U \cap X$. So $|P(h(x))| \leq$ const $\cdot|x|^{\theta / \max _{j} r_{j}}$ for $x \in U \cap X$ and so the lemma is proved.

We finish the proof of the corollary by observing that by the Lemma together with Proposition (5.2) we have (since $\left.v\left(h_{1} h_{2}\right)=v\left(h_{1}\right)+v\left(h_{2}\right)\right)$

$$
q_{j} \operatorname{ord}_{0} f_{j}+\operatorname{ord}_{0}\left(H_{j}(F, G)\right) \geq \operatorname{deg}_{0} A q \operatorname{ord}_{0} g \prod_{i=1}^{k} q_{i} \operatorname{ord}_{0} f_{i}
$$

and since $P_{G}(F, G) \equiv 0$, it suffices to take $h_{j}:=-H_{j}(F, G)$.

(5.4) Lemma. Let $A$ be an analytic set of pure dimension $k \geq 1$ in an open set $\Omega \subset \mathbb{C}^{m}$ and suppose that $T_{0}^{\text {Zar }} A=\mathbb{C}^{m}$ (i.e. $A$ is minimally embedded in $\mathbb{C}^{m}$ at zero). Then coordinates $x_{j}$ of $\mathbb{C}^{m}$ can be chosen in such a way that $\operatorname{ord}_{0}\left(\left.x_{j}\right|_{A}\right)=1$ for $j=1, \ldots, m$.

Proof. For the generic choice of coordinates the set $A$ has proper projection at zero on any $k$-dimensional coordinate plane and each such projection realizes $\operatorname{deg}_{0} A$. We will show that $\operatorname{ord}_{0}\left(\left.x_{1}\right|_{A}\right)=1$.

Consider the projection $\pi$ on the first $k$ coordinates and take a line $l \in U$, where $U$ is the set from Proposition (4.4). The line can be chosen so that $l \cap\left\{x_{1}=0\right\}=\{0\}$. Then, in the notation of Proposition (4.4), $S_{l}$ is transversal to $\left\{x_{1}=0\right\}$. Therefore for any irreducible component $\Gamma \subset S_{l}$ and its Puiseux parametrization $\gamma$ one has $\operatorname{ord}_{0}\left(x_{1} \circ \gamma\right)=\operatorname{ord}_{0} x_{1} \cdot \operatorname{ord}_{0} \gamma$ and so by Proposition (4.4) we obtain $\operatorname{ord}_{0}\left(\left.x_{1}\right|_{A}\right)=1$.

All this leads to the following estimate of the Łojasiewicz exponent: 
(5.5) Theorem. Let $A$ be an analytic set of pure dimension $k \geq 1$ in an open set $\Omega \subset \mathbb{C}^{m}$ and let $f \in \mathcal{O}_{\mathrm{c}}\left(A, \mathbb{C}^{k}\right)$ be such that $f^{-1}(0)=\{0\}$. Then

$\max _{j=1}^{k} \operatorname{ord}_{0} f_{j} \leq \mathcal{L}(f ; 0) \leq[D(f)]^{k-1}\left(m_{0}(f)-\operatorname{deg}_{0} A \prod_{j=1}^{k} \operatorname{ord}_{0} f_{j}\right)+\max _{j=1}^{k} \operatorname{ord}_{0} f_{j}$, where $D(f)=\min \left\{q \in \mathbb{N} \mid q \operatorname{ord}_{0} f_{j} \in \mathbb{N}, j=1, \ldots, k\right\}$ is the smallest common denominator of the orders. Note that $D(f) \leq\left(\operatorname{deg}_{0} A\right)$ !.

Proof. We start with the proof of the leftmost inequality. Let $\max _{j} \operatorname{ord}_{0} f_{j}$ $=\operatorname{ord}_{0} f_{k}$. Since $\bigcap_{j} f_{j}^{-1}(0)$ is a proper intersection, one can check that $\left\{f_{1}=\cdots=f_{k-1}=0\right\}$ is a pure one-dimensional germ near zero. Let $\gamma$ be a Puiseux parametrization of any of its components. Then by Theorem (3.2) (and since $f_{k} \circ \gamma \not \equiv 0$ ) we have

$$
\mathcal{L}(f ; 0) \geq \mathcal{L}(f \circ \gamma ; 0)=\frac{\operatorname{ord}_{0}\left(f_{k} \circ \gamma\right)}{\operatorname{ord}_{0} \gamma} .
$$

On the other hand, $\operatorname{ord}_{0}\left(f_{k} \circ \gamma\right) / \operatorname{ord}_{0} \gamma \geq \operatorname{ord}_{0} f_{k}$, which completes the proof.

We now proceed to proving the second inequality. Similarly to the holomorphic case it is a more or less direct consequence of (5.3). We may assume that $A$ is minimally embedded in $\mathbb{C}^{m}$ at zero and coordinates are chosen by means of Lemma (5.4).

Let $d:=D(f)$. For $j \in\{1, \ldots, k\}$ let $G_{j}$ be the $j$ th coordinate function $x_{j}$ in $\mathbb{C}^{m}$ restricted to $A$. Set also $F:=\left(f_{1}^{d}, \ldots, f_{k}^{d}\right)$ and observe that $\mathcal{L}(F ; 0)=$ $d \mathcal{L}(f ; 0)$. Then we have the identity $G_{j}^{m_{0}(F)}=\sum_{i=1}^{k} h_{i, j} F_{i}$ with $h_{i, j} \in \mathcal{O}_{\mathrm{c}}(A)$ such that

$$
\operatorname{ord}_{0} h_{i, j} \geq \operatorname{deg}_{0} A \operatorname{ord}_{0} G_{j} \cdot d^{k} \prod_{i=1}^{k} \operatorname{ord}_{0} f_{i}-d \underset{i=1}{k} \underset{\max }{k} \operatorname{ord}_{0} f_{i} .
$$

However, $\operatorname{ord}_{0}\left(\left.x_{j}\right|_{A}\right)=1$ and so in a nbhd of zero in $A$ we have

$$
\left|x_{j}\right|^{d^{k} m_{0}(f)} \leq \mathrm{const} \cdot|x|^{\operatorname{deg}_{0} A d^{k} \prod_{i=1}^{k} \operatorname{ord}_{0} f_{i}-d \max _{i=1}^{k} \operatorname{ord}_{0} f_{i}}|F(x)|,
$$

which yields the desired inequality.

(5.6) Remark. If $A$ is an irreducible analytic curve, we get exactly the statement of Theorem (3.2) since in this case $m_{0}(f)=\operatorname{ord}_{0}(f \circ \gamma)(\gamma$ being injective). Furthermore, if $A$ is an open set in $\mathbb{C}^{k}$, we have exactly the statement of [P2].

Moreover, applying the methods of [S] one verifies that the result is still valid if the target space is of dimension $n>k$. Under the assumption that $\operatorname{ord}_{0} f_{1} \leq \cdots \leq \operatorname{ord}_{0} f_{n}$ it reads exactly as stated in the theorem (i.e. we take into account only the first $k$ orders). 
Acknowledgements. It is a pleasure to express my gratitude to Prof. Piotr Tworzewski for introducing me to the subject and many valuable remarks. I am also deeply indebted to Prof. Alain Yger for very stimulating discussions and his kind help. Most of this work took shape during my stay at the University Bordeaux 1 whose staff deserves my warmest thanks. And above all I would like to thank my wife Anna for her constant patience and love which are the best support for my work.

\section{References}

[ATW] R. Achilles, P. Tworzewski and T. Winiarski, On improper isolated intersection in complex analytic geometry, Ann. Polon. Math. 51 (1990), 21-36.

[Chd] J. Chądzyński, On the order of an isolated zero of a holomorphic mapping, Bull. Polish Acad. Sci. Math. 31 (1983), 121-128.

[Ch] E. M. Chirka, Complex Analytic Sets, Kluwer, 1989.

[Cg] E. Cygan, Intersection theory and separation exponent in complex analytic geometry, ibid. 69 (1998), 287-299.

[CgT] E. Cygan and P. Tworzewski, Proper intersection multiplicity and regular separation of analytic sets, ibid. 59 (1994), 293-298.

[Dr] R. N. Draper, Intersection theory in analytic geometry, Math. Ann. 180 (1969), 175-204.

[Ł1] S. Łojasiewicz, Ensembles semi-analytiques, preprint I.H.E.S., 1965.

[Ł2] - Introduction to Complex Analytic Geometry, Birkhäuser, Basel, 1991.

[P1] A. Płoski, Une évaluation pour les sous-ensembles analytiques complexes, Bull. Polish Acad. Sci. Math. 31 (1983), 259-262.

[P2] - Sur l'exposant d'une application analytique I, ibid. 32 (1984), 669-673.

[P3] - Multiplicity and the Eojasiewicz exponent, in: Singularities, Banach Center Publ. 20, PWN, Warszawa, 1988, 353-364.

[Sh] N. V. Shcherbina, Pluripolar graphs are holomorphic, preprint 3/2003, Chalmers/Göteborg Univ., 2003.

[S] S. Spodzieja, Multiplicity and the Eojasiewicz exponent, Ann. Polon. Math. 73 (2000), 257-267.

[T1] P. Tworzewski, Isolated intersection and regular separation of analytic sets, ibid. 57 (1993), 213-219.

[T2] -, Intersection theory in complex analytic geometry, ibid. 62 (1995), 177-191.

[Wh] H. Whitney, Complex Analytic Varieties, Addison-Wesley, 1972.

Institute of Mathematics

Jagiellonian University

Reymonta 4

30-059 Kraków, Poland

E-mail: denkowsk@im.uj.edu.pl
Institut de Mathématiques de Bordeaux Université Bordeaux 1 351, cours de la Libération 33405 Talence, France

E-mail: denkowsk@math.u-bordeaux1.fr 